\title{
Authors' reply re: Universal screening versus risk-based protocols for antibiotic prophylaxis during childbirth to prevent early-onset Group B streptococcal disease: A systematic review and meta-analysis. (Response to BJOG-20-0671)
}

\author{
Geke Hasperhoven ${ }^{1}$, Salwan Al-Nasiry², Vincent Bekker ${ }^{3}$, Eduardo Villamor ${ }^{1}$, and Boris \\ Kramer $^{1}$ \\ ${ }^{1}$ Maastricht University Medical Centre+ \\ ${ }^{2}$ Maastricht UMC \\ ${ }^{3}$ Leiden University Medical Center
}

May 6, 2020

Dear Editor,

We would like to thank Dr. Lamont and colleagues for their interesting commentary. ${ }^{1}$ The authors point out very pressing topics that deserve more attention than we could provide in the original manuscript.

They expressed concerns in their letter towards the heterogeneity of the data that were used in our metaanalysis. If incidences differ between regions and populations, absolute numbers on colonisation and neonatal infections might not be comparable. However, new policies were implemented in various settings, where differences in outcomes were measured under the same local circumstances. So as rightly mentioned by Lamont et al, data from the US cannot and should not be used as a blueprint for other parts of the world. ${ }^{1}$ Yet the general trend in developed countries that we observed, might shed some new light on the respective effectiveness of the strategies currently available.

We further agree with the authors that the use of rapid PCR testing should be investigated for its clinical benefits, sensitivity and specificity. This is even more pressing as the colonisation of GBS is transient, as addressed through the statistics provided by Lamont et al. ${ }^{1}$ Transient colonisation leads to problems of missing cases when using culture in the screening policies. However missed cases occur in risk-based policies as well. We extracted data from studies in the review, which showed missed cases to be $41 \%$ of EOGBS cases in the risk-based group compared to $24 \%$ in the screening group (weighted mean). ${ }^{2}$

When it comes to preterm birth, innovative ways are needed without doubt, as EOGBS sepsis in this group of infants results in a much higher case-fatality rate compared to term infants. ${ }^{3}$ Besides, little data is available on the role of GBS infection as the cause of preterm birth. Most screening-protocols suggest all women with impeding preterm birth receive IAP. More targeted prophylaxis might reduce overtreatment and potentially reduce the occurrence of late-onset sepsis as early antibiotic exposure is a risk-factor for NEC and late-onset sepsis. Once timely PCR-testing is fully validated and implemented, important steps could be made to understand and to protect this group.

Lamont et al. also suggest focussing on the development of a vaccine to tackle these complex pathological processes in pregnancies at risk of adverse outcomes. ${ }^{1}$ Unfortunately, such a vaccine is not yet available, and the issues concerning IAP will also be present in vaccine implementation, e.g. the selection of a population and timing of prophylaxis/immunisation. ${ }^{4}$ 
Neither screening nor risk-based policies are perfect. As it is unclear when and for whom PCR testing or vaccination will be available, it is worth identifying the most effective policy to protect infants from invasive GBS infections. Currently, the GBS3 trial is being conducted in the UK. ${ }^{5}$ Hopefully, there soon will be clinical data available on effectiveness and cost-effectiveness, to further help the discourse on this important topic.

\section{References}

1. Lamont R, Joergensen SS, Vinter C. Re: Universal screening versus risk-based protocols for antibiotic prophylaxis during childbirth to prevent early- onset Group B streptococcal disease: A systematic review and meta-analysis. (First comment letter. Reference to be added).

2. Hasperhoven GF, Al-Nasiry S, Bekker V, Villamor E, Kramer BW. Universal screening versus riskbased protocols for antibiotic prophylaxis during childbirth to prevent early-onset Group B streptococcal disease: a systematic review and meta-analysis. Vol. 127, BJOG: An International Journal of Obstetrics \& Gynaecology. 2020;127(6). doi: 10.1111/1471-0528

3. Stoll BJ, Hansen NI, Sanchez PJ, Faix RG, Poindexter BB, Van Meurs KP, et al. Early Onset Neonatal Sepsis: The Burden of Group B Streptococcal and E. coli Disease Continues. Vol. 127, Pediatrics. 2011 May 1;127(5):817-26. doi: 10.1542/peds.2010-2217

4. Kenchington AL, Lamont RF. Group B streptococcal immunisation of pregnant women for the prevention of early and late onset Group B streptococcal infection of the neonate as well as adult disease. Vol. 16, Expert Review of Vaccines. Taylor and Francis Ltd; 2017. p. 15-25. doi: 10.1080/14760584.2016.1209113

5. Walker K, Gray J, Petrou S, Ayers S, Ogollah R, Ojha S, et al. The clinical and cost-effectiveness of testing for Group B Streptococcus: a cluster randomised trial with economic and acceptability evaluations (GBS3). Vol. 126, BJOG : an international journal of obstetrics and gynaecology. 2019;126(S1). 